\title{
Nozzleless fabrication of oil-core biopolymeric microcapsules by the interfacial gelation of pickering emulsion templates
}

\begin{abstract}
Ionotropic gelation has been an attractive method for the fabrication of biopolymeric oil-core microcapsules due to its safe and mild processing conditions. However, the mandatory use of a nozzle system to form the microcapsules restricts the process scalability and the production of small microcapsules $(<100 \mu \mathrm{m})$. We report, for the first time, a nozzleless and surfactantfree approach to fabricate oil-core biopolymeric microcapsules through ionotropic gelation at the interface of an $\mathrm{O} / \mathrm{W}$ Pickering emulsion. This approach involves the self-assembly of calcium carbonate $(\mathrm{CaCO} 3)$ nanoparticles at the interface of $\mathrm{O} / \mathrm{W}$ emulsion droplets followed by the addition of a polyanionic biopolymer into the aqueous phase. Subsequently, $\mathrm{CaCO} 3$ nanoparticles are dissolved by $\mathrm{pH}$ reduction, thus liberating $\mathrm{Ca}(2+)$ ions to cross-link the surrounding polyanionic biopolymer to form a shell that encapsulates the oil droplet. We demonstrate the versatility of this method by fabricating microcapsules from different types of polyanionic biopolymers (i.e., alginate, pectin, and gellan gum) and water-immiscible liquid cores (i.e., palm olein, cyclohexane, dichloromethane, and toluene). In addition, small microcapsules with a mean size smaller than $100 \mu \mathrm{m}$ can be produced by selecting the appropriate conventional emulsification methods available to prepare the Pickering emulsion. The simplicity and versatility of this method allows biopolymeric microcapsules to be fabricated with ease by ionotropic gelation for numerous applications.
\end{abstract}

Keyword: Pickering emulsion; Biopolymer; Core-shell; Ionotropic gelation; Microcapsules 